\title{
New Design of a Microstrip-Fed Printed Slot UWB Antenna with Hilbert Fractal Defected Ground Structure
}

\author{
Khadidja Krim, Mehadji Abri, Hadjira Badaoui, Samira Fersiti,Fatima Zahra Mahieddine \\ Krim.khadidja@gmail.com, abrim2002@yahoo.fr, elnbh@yahoo.fr
}

STIC Laboratory, Department of Telecommunication, Faculty of Technology,University of Tlemcen, Algeria.

\begin{abstract}
In this paper, we propose originals structures of monopole microstrip antennas for UWB applications with Hilbert curve Defected Ground Space (DGS) structure. These Antennas are made of two different shapes of slot inside a square patch, fed by a $50 \Omega$ microstrip line on a FR4 dielectric substrate. A 3rd iteration of the Hilbert space filing curve is cut out from a partial ground plane. These antennas have a wide bandwith varying from $3.21 \mathrm{GHz}$ to $13.64 \mathrm{GHz}$ and from $3.18 \mathrm{GHz}$ to $14.32 \mathrm{GHz}$ for $\mathrm{S} 11<-10 \mathrm{~dB}$. Details of the two antenna designs approach and simulated results are presented and discussed in this paper.
\end{abstract}

Keywords: Antenna, Hilbert Fractal shape, Ultra Wide Band Antenna, Microstrip patch antenna.

\section{Introduction}

Defects integrated on the ground plane or slots of microwave planar circuits are colled Defected Ground Structure. DGS is used as an emerging method for improving the various parameters of microwave circuits, like: narrow bandwidth, low gain, cross-polarization ...etc.[1].

developments in wireless communication system srequired additional challenges on circuit designers and microwave antenna to ceate new designs miniaturized and multiband [2],[3].

The pionee work of Mandelbrot [4] had stimulated microwave circuits and antenna designers in 1975, who were trying to realize miniaturized circuits and components, to look for solutions by exploring different fractal geometries [5] derived from the latin word "fractus", signifying "broken" or "fractured". The object was to describe nature's geometry and classify complex geometries that were designed with an iterative procedure [6], whose dimensions were not whole numbers [7].

Fractal curves have a unique property that, after an infinite number of iterations, their length becomes infinite although the entire curve fits into the finite area. This property can be used in order to minaturize microstrip antennas, resonators, and filters. Because of technology limitations [8-9], fractal curves are not physically realizable. Pre-fractals, fractal curves with finite order, are used rather [10], [11].

Hilbert, Peano, and Gosper space-filling curves have stimulated the researchers to realize miniatures antennas with multiple resonances [12].

In this paper two new types of monopole microstrip antennas are proposed, Obtained by the third iteration of the Hilbert fractal curve cut out of a partial ground plane, and two shapes of slot in a square patch. 
The proposed antennas are characterized by a wide bandwith with a stable direction of radiation.

\section{Description of the proposed antennas}

Hilbert curve is a continuous fractal space-filling curve first described by the German mathematician David Hilbert in 1891, as a variant of the space-filling Peano curves discovered by Giuseppe Peano in 1890.

Various iteration stages of Hilbert curves are shown in Fig.1 [13].

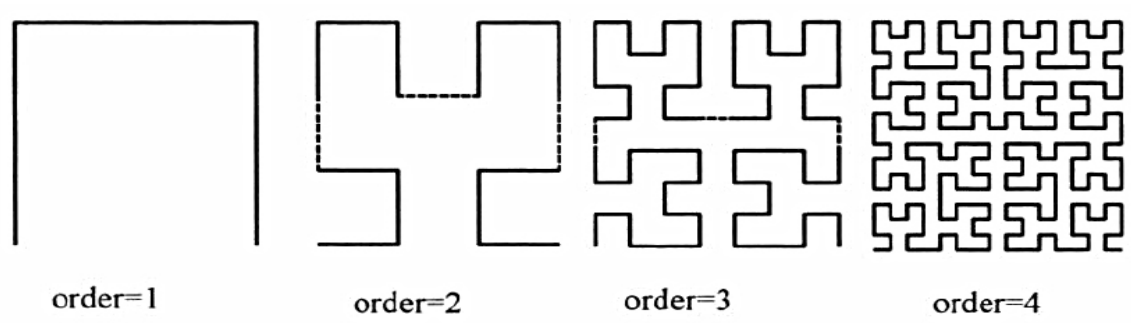

Fig. 1. First four fractal iterations for the Hilbert curve geometry.

In this paper, we propose two monopole antenna structures composed of different shapes of slots inside a square patch.

These antennas were printed and fed by a $50 \Omega$ microstrip transmission line on a FR4 dielectric substrate with thickness $\mathrm{h}=1.54 \mathrm{~mm}$, and a loss tangent of 0.018 and dielectric constant $\varepsilon r=4.3$.

The third iteration of Hilbert curve slot was constructed on the ground plane side of a substrate measuring $33 \mathrm{~mm}$ by $33 \mathrm{~mm}$.

Study of these antennas was conducted using CST Microwave Studio Software.

\section{Simulation Results}

A "I" shape of slot inside a square patch is simulated with third iteration Hilbert curve placed on the center of the partial ground plane as shown in fig. 2 (a) and (b). The patch is modified by inserting small steps (Fig. 2 (a)).

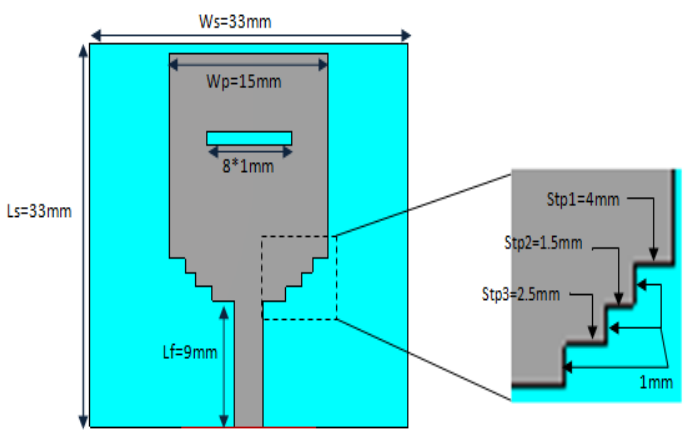

(a)

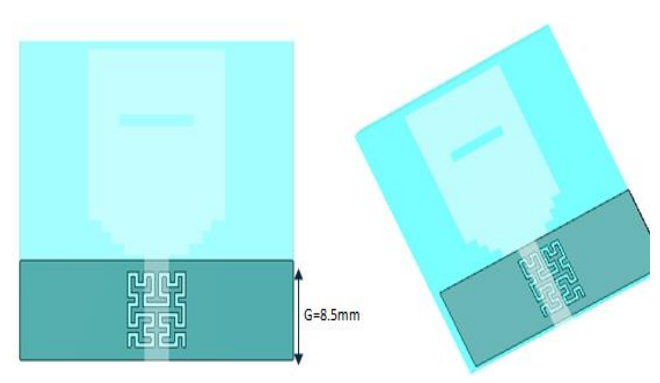

(b)

Fig. 2. (a) Rectangle Monopole Antenna geometry. (b) Ground Plane structure for the first antenna. 
Fig. 3 illustrates the reflection coefficient S11 of the antecedent designed antenna from 1 $\mathrm{GHz}$ to $16 \mathrm{GHz}$.

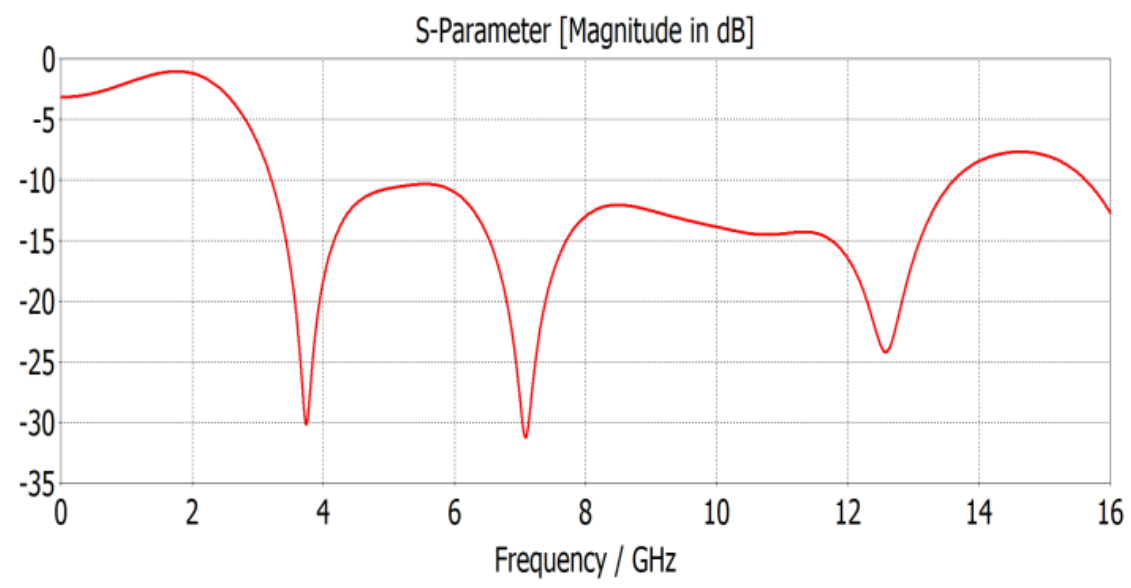

Fig. 3. Simulated Reflection coefficient versus frequency of the first designed antenna.

We observe that the antenna resonance is correctly predicted by three peaks $-31.64 \mathrm{~dB}$, $30.08 \mathrm{~dB}$ and $-24.43 \mathrm{~dB}$ at $3.74 \mathrm{GHz}, 7.88 \mathrm{GHz}$ and $12.58 \mathrm{GHz}$ respectively. The band with (with the definition of $-10 \mathrm{~dB}$ ) for the designed antenna is from $3.21 \mathrm{GHz}$ to $13.64 \mathrm{GHz}$, which may cover the UWB frequency range.

The 3D radiation patterns of the first designed antenna structure at some frequency points are illustrated in Fig.4. It shows acceptable stability of the radiation. It's shown that gain increases for higher frequencies. It's around $2.45 \mathrm{dBi}, 3.76 \mathrm{dBi}$, and $5.64 \mathrm{dBi}$ at: $3.74 \mathrm{GHz}$, $7.33 \mathrm{GHz}$, and $12.33 \mathrm{GHz}$ respectively.

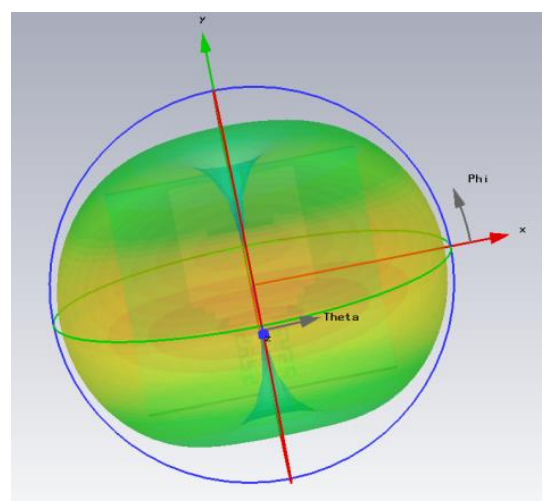

$f=3.74 \mathrm{GHz}$

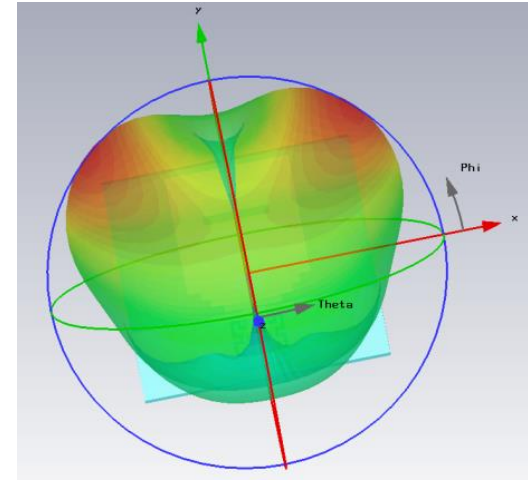

$f=7.45 \mathrm{GHz}$ 


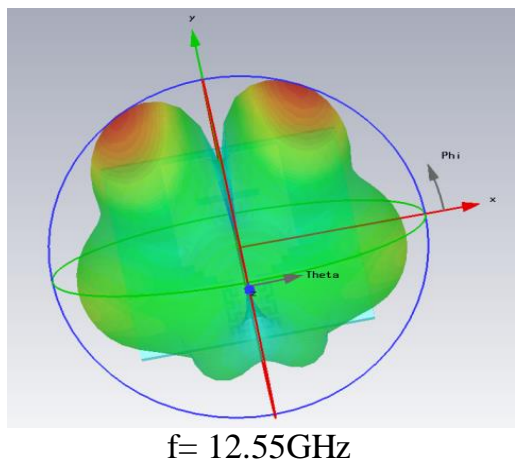

Fig. 4. Simulated 3D radiation patterns of the first antenna at some frequencies.

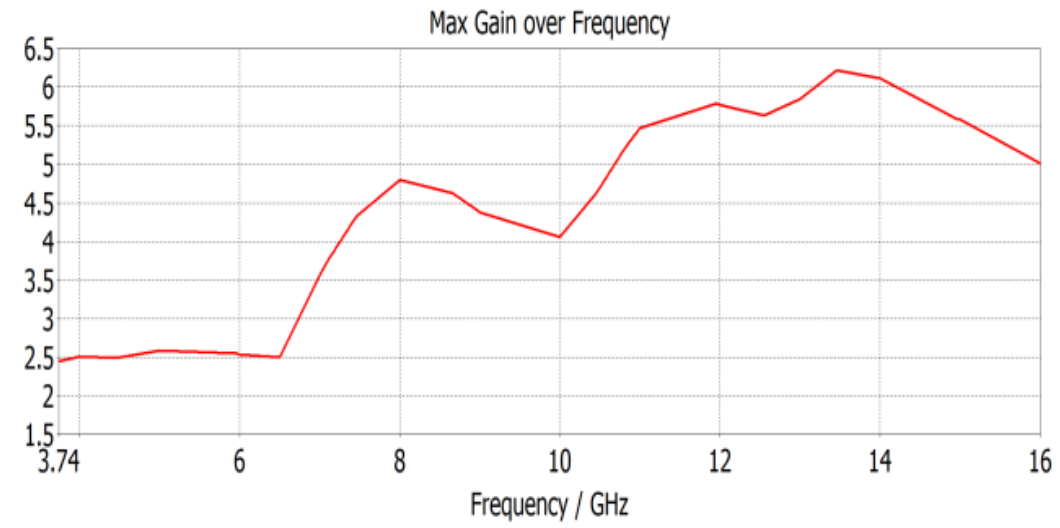

Fig. 5. Variation of simulated Gain in $\mathrm{dBi}$ against frequency for the first antenna.
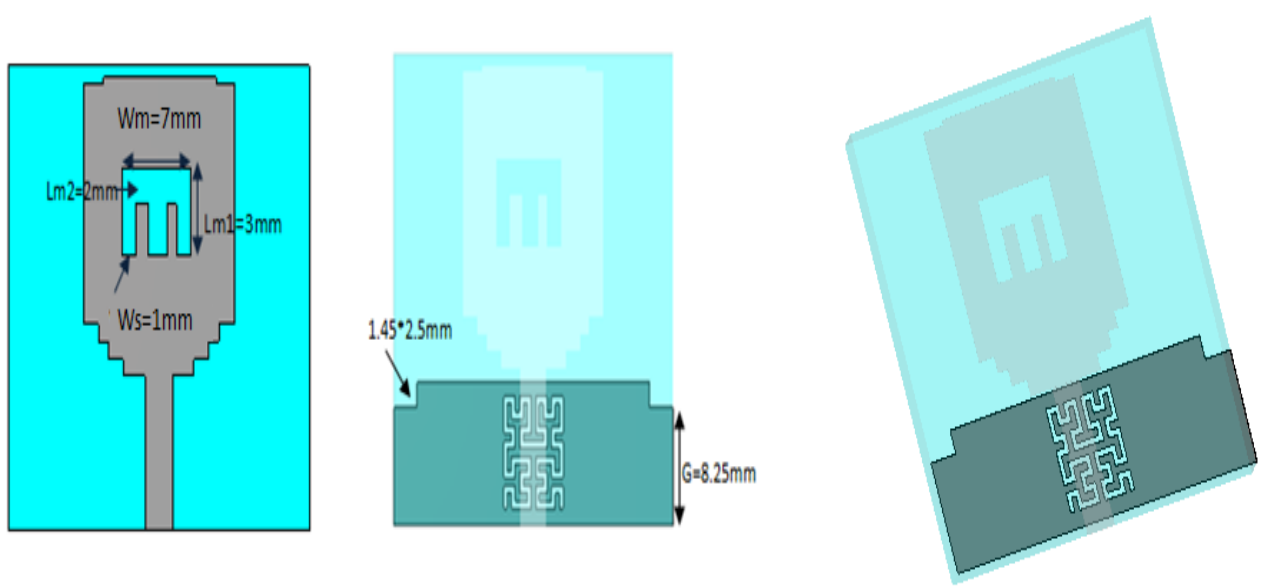

Fig. 6. Geometry of the second proposed monopole Antenna. 
For this antenna we use Another shape of slot (E shape), and te ground is modified by inserting two small steps as shown in fig. 6.

Fig. 7 shows the simulated S11 plot of the second designed antenna, the band with (with the definition of $-10 \mathrm{~dB}$ ) is from $3.18 \mathrm{GHz}$ to $14.32 \mathrm{GHz}$, and cover the UWB frequency range.

Three peaks are remarked: $-25.82 \mathrm{~dB},-26.15 \mathrm{~dB}$, and and $-17.02 \mathrm{~dB}$ at $3.71 \mathrm{GHz}, 6.97$, and $13.05 \mathrm{GHz}$.

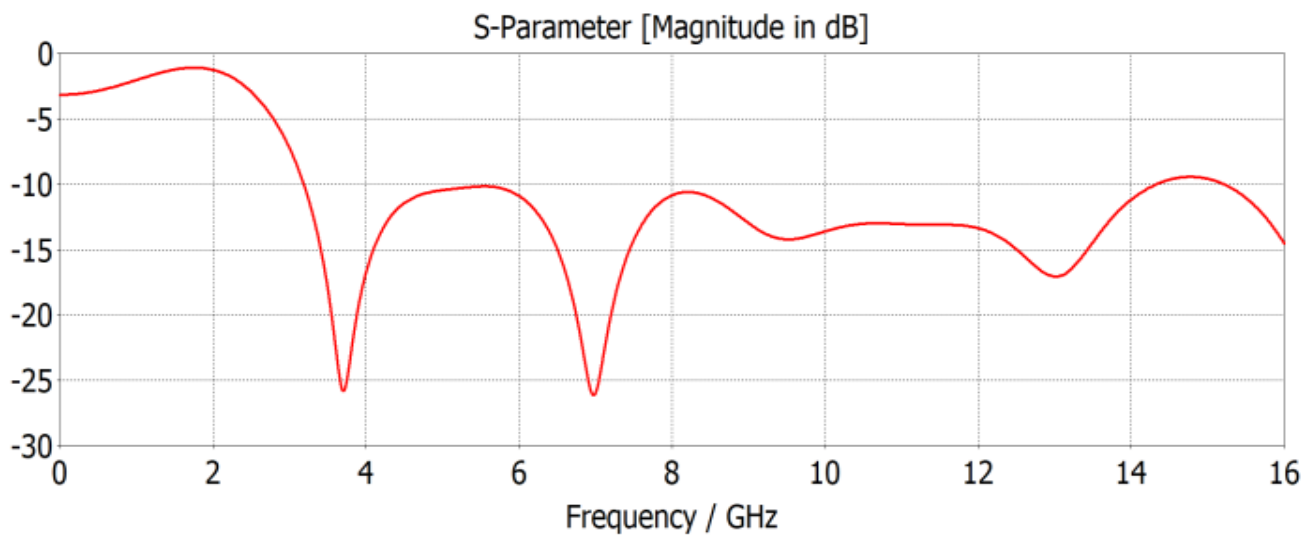

Fig. 7. Simulated Reflection coefficient versus frequency of the second designed antenna.

The 3D radiation patterns of the antenna structure at some frequency points are illustrated in Fig. 8. It shows acceptable stability of the radiation.

It's shown that gain increases for higher frequencies. It's around $2.5 \mathrm{dBi}, 4.51 \mathrm{dBi}$, and $5.14 \mathrm{dBi}$ at : $3.91 \mathrm{GHz}, 8.25 \mathrm{GHz}$, and $12.25 \mathrm{GHz}$ respectively.

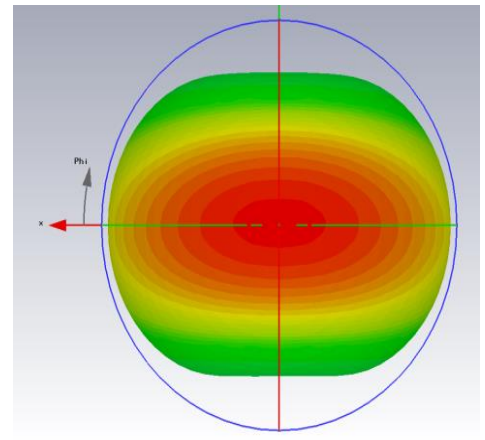

$f=3.91 \mathrm{GHz}$

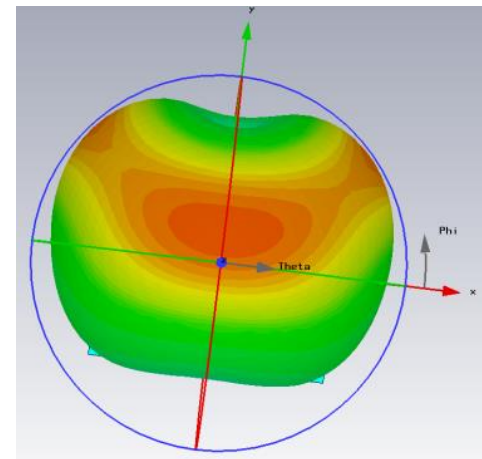

$f=6.5 \mathrm{GHz}$ 


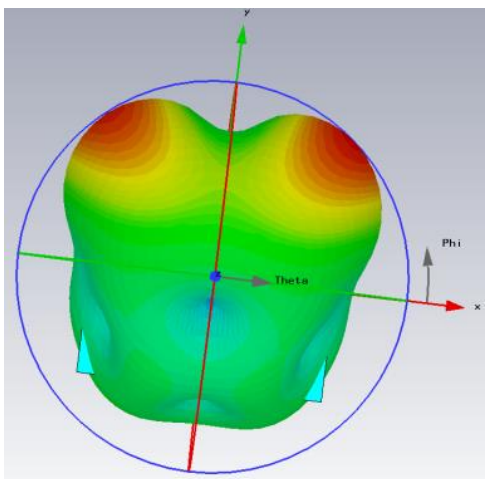

$f=8.25 \mathrm{GHz}$

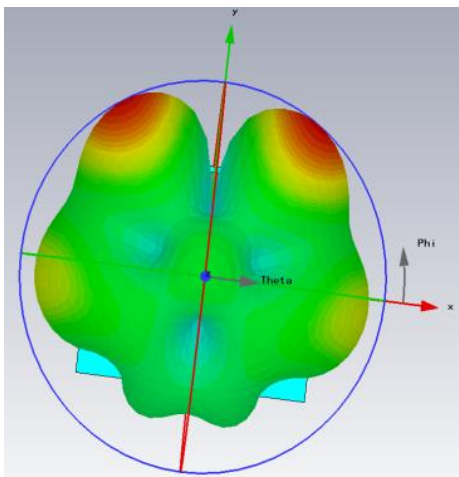

$f=12.5 \mathrm{GHz}$

Fig. 8. Simulated 3D radiation patterns of the second antenna at some frequencies.

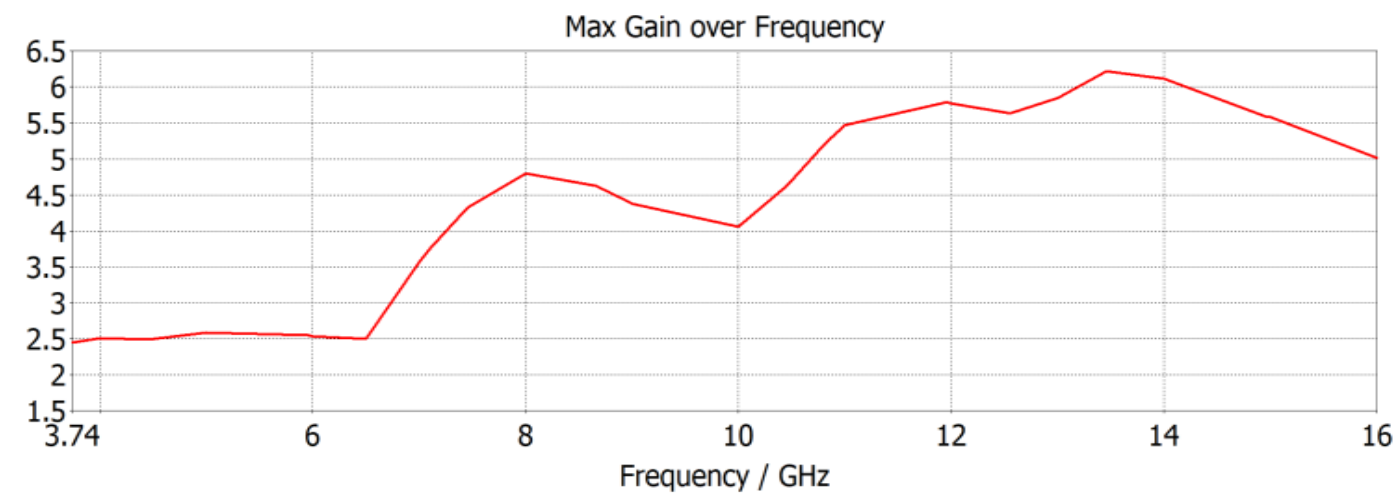

Fig. 9. Variation of simulated Gain in dBi against frequency for the second antenna.

\section{Conclusion}

In this paper, two new monopole microstrip antennas are proposed; composed of Hilbert curve partial defected ground plane and two different shapes of slot inside a square patch. These antennas have been simulated by SCT microwave studio software.

The simulations results show that both of the proposed antennas offer a wide band with able to be used in UWB applications. 


\section{References}

[1] Mukesh Kumar Khandelwal, Binod Kumar Kanaujia, Sachin Kumar. 'Defected Ground Structure: Fundamentals, Analysis, and Applications in Modern Wireless Trends, International

Journal of Antennas and Propagation'

Volume 2017, Article ID 2018527, 22 pages.

[2] S. Didouh, M. Abri, and F. T. Bendimerad, 'Corporate-Feed Multilayer Bow-Tie Antenna Array Design Using a Simple Transmission Line Model', Modelling and Simulation in Engineering Volume 2012 (2012), Article ID 327901, 8 pages.

[3] Mohammed Moulay, Mehadji Abri, and Hadjira Abri Badaoui, 'Quad-Band Bowtie Antenna Design for Wireless Communication System Using an Accurate Equivalent Circuit Model', International Journal of Microwave Science and Technology, Article ID 637607, 2015.

[4] B. B. Mandelbrot, 'The Fractal Geometry of Nature' (W. H. Freeman, 1983).

[5] Jawad K. Ali, 'Microstrip-Fed Printed Slot Antennas Based on Hilbert-Type Space- Filling Curves for Wireless communication Systems', 2013.

[6] K Falconer, 'Fractal Geometry: Mathematical foundations and applications', John Wiley and Sons, Chichester, 1990.

[7] M. ABRI, H. DIB and A. S. E. GHARNAOUT, 'Accurate Model for Single Bow-Tie Antenna Design', INTERNATIONAL JOURNAL OF MICROWAVE AND OPTICAL TECHNOLOGY, VOL.6 NO.5, pp. 294-300, 2011.

[8] M. Abri, N. Belgacem and W. Belgacem 'New GSM, DCS and GSM/DCS PIFA Antennas Designs for Wireless Networks applications', International Journal of Information \& Network Security (IJINS) Vol.2, No.4, August 2013, pp. 305 310 ISSN: 2089-3299, 2013.

[9] M.Moulay, M.Abri, 'Bowtie antennas design for bluetooth/wimax/wifi application', International journalof microwave and optical technology 9 (4), 282-287,2014.

[10] J. P. Gianvittorio, 'Fractals, MEMS, and FSS Electromagnetic Devices: Miniaturization and Multiple Resonances', Ph.D. dissertation, University of California, Los Angeles, 2003.

[11] K. J. Vinoy, K. A. Jose, V. K. Varadan, and V. V. Varadan, 'Hilbert Curve Fractal Antenna: A small Resonant Antenna for VHF/UHF Applications', Microwave Opt. Technol. Lett., Vol. 29(Issue 3):215-219, May 2001.

[12] S. R. Best, 'A Comparison of the Performance Properties of theHilbert Curve Fractal and Meander Line Monopole Antennas', Microwave Opt. Technol. Lett., Vol. 35(Issue 11): 258-262, November 2002.

[13] J. Anguera, C. Puente, E. Martinez, and E. Rozan, 'The FractalHilbert Monopole: A TwoDimensional Wire’, Microwave Opt.Technol. Lett., Vol. 36(Issue 1): 102-104, January 2003. 